\title{
OTIMIZAÇÃO DO PROBLEMA DE CORTE UNIDIMENSIONAL: UM ESTUDO DE CASO NA INDÚSTRIA PAPELEIRA
}

\author{
Bruno Oliveira (UTFPR, Brasil) brunoengenharia@outlook.com \\ Rodrigo Salvador (UTFPR, Brasil) salvador.rodrigors@gmail.com \\ Vanina Macowski Durski Silva (UFSC, Brasil) vanina.durski@ufsc.br
}

\begin{abstract}
Resumo: Com o acirramento da competitividade e a intensa e constante mudança tecnológica que o mercado vem sofrendo, a utilização de métodos que busquem otimizar a produção, quer seja no aspecto econômico, ambiental ou outro, se apresenta mais do que apenas um fator diferencial de competitividade. Nesta ótica o uso de ferramentas de pesquisa operacional na solução de problemas e otimização de processos é de extrema importância. Assim, este trabalho, um estudo de caso na indústria papeleira, objetiva minimizar a quantidade de resíduos gerada nas atividades de corte do processo de produção de bobinas de papel cartão de uma matriz (bobina) jumbo, em um mês de operação em uma unidade de uma companhia do ramo papeleiro da região dos Campos gerais, por meio do uso de métodos de programação linear. Foi utilizada para solução do problema de corte unidimensional, inicialmente a modelagem matemática, construída com o auxílio da ferramenta LINGO. A solução ótima encontrada para o cenário estabelecido foi uma sobra de $685.000 \mathrm{~m}$ (e um comprimento fixo de $10.000 \mathrm{~m}$ ), atendendo a todas as restrições impostas pelo modelo.

Palavras-chave: Otimização. Problema de Corte. Resíduos. Indústria Papeleira.
\end{abstract}

\section{Introdução}

Com o desenvolvimento tecnológico, a globalização e a cada vez mais acirrada competitividade, além do aumento da preocupação com um desenvolvimento sustentável, o desenvolvimento e aperfeiçoamento de novos métodos em busca da melhoria de processos se torna fator diferencial dentro da estratégia de planejamento e ação de uma empresa.
A busca pelo aumento da eficiência de cada parcela do processo produtivo é inerente à sede de evolução de toda a cadeia de produção. Num aspecto geral, cada avanço contribui para uma progressão rumo a um aperfeiçoamento global (KAMIMURA; PAES; OLIVEIRA, 2012; LUZ et al., 2012).

Dentro deste contexto, pode-se destacar como ferramenta auxiliadora a pesquisa operacional, que consiste em uma abordagem científica com a finalidade de prever e comparar estratégias e decisões alternativas, de modo a alcançar o 
aperfeiçoamento da produção, proporcionando a otimização do processo envolvido por meio de uma simplificação do problema real a partir de um modelo que o represente (POLDI; ARENALES, 2006).

Em face ao exposto, o objetivo deste trabalho é encontrar o plano de produção que minimize a quantidade de resíduos gerada nas atividades de corte do processo de produção de bobinas de papel cartão, em um mês de operação de uma unidade de uma empresa do ramo papeleiro, utilizando programação linear (PL).

Este estudo justifica-se pela necessidade do cuidado com o meio. Bem como com os aspectos econômicos da produção, que levam à discussão de melhores maneiras de praticá-la, de modo que a empresa possa desfrutar de uma prática sustentável, reduzindo os impactos inerentes à atividade desenvolvida e podendo, ao mesmo tempo, obter ganhos econômicos e de marketing.

Assim, evidencia-se a necessidade de planejar a produção visando adaptar o material primário às demandas dos clientes. As sobras das atividades de corte não sofrem simples descarte. Devido às suas características são incorporadas, em um novo ciclo da produção, à camada intermediária de um novo lote de papel cartão.

Neste sentido, de acordo com Cherry, Além Junior e Silva (2010), o aproveitamento das sobras é prática real em muitas organizações, principalmente quando a matéria-prima se mostra como uma parcela significativa do custo total produto final. Observa-se, ainda, grande importância da característica sustentável desta prática, tendo influência no âmbito econômico, além de social e ambiental. Como afirmam Silveira Júnior, Pinheiro e Thomaz (2004), as proporções alcançadas por tais perdas, as sobras das operações de corte, podem ser gigantescas, a priori em detrimento do atendimento da demanda, sendo importantes fatores na geração do custo final dos produtos e mesmo das operações de produção. Além do exposto, a preocupação com os parâmetros gerais da produção englobam não somente aspectos econômicos, mas também evidenciam preocupação com a geração de resíduos e consequentes desperdícios de matérias-primas como energia e outros, que são responsáveis, muitas vezes, pela depreciação de fontes renováveis e não renováveis.

Frente a este contexto, o objetivo deste trabalho é encontrar o plano de produção que minimize a quantidade de resíduos gerada nas atividades de corte, da produção de bobinas de papel cartão, em um mês de operação da unidade Monte Alegre das indústrias Klabin, utilizando programação linear (PL).

Acordando com esta perspectiva, este artigo está organizado em 6 seções. A metodologia empregada para a realização do mesmo é apresentada na sequência, seguida da seção 2, onde é apresentado um breve contexto a respeito das características de produção na unidade física utilizada para a realização deste estudo. A seção 3 apresenta o conceito de Pesquisa Operacional, mais especificamente a definição do problema de corte unidimensional, conceito empregado na 
modelagem do problema de estudo. Tal problema é apresentado e contextualizado na seção 4. Dando continuidade, a seção 5 apresenta a modelagem matemática proposta para o problema em estudo, as análises e resultados obtidos, seguida da última seção, referente às considerações finais, e então as referências bibliográficas.

\section{Metodologia}

A abordagem metodológica utilizada para a realização deste trabalho constitui-se de um estudo de caso, tendo como foco a unidade fabril de Monte Alegre das Indústrias Klabin. Em visita à unidade, com o acompanhamento de um responsável técnico pela área, tendo o conhecimento de todo o funcionamento das instalações. Identificou-se um ponto potencial de melhoria na etapa de corte de bobinas jumbo e, levando em conta os aspectos econômicos, pelo atendimento da demanda e estoque, e de sustentabilidade, pela minimização dos resíduos. Formulou-se um modelo de programação linear, o qual foi modelado com o auxílio da ferramenta LINGO, para a solução do problema e descoberta da solução ótima.
Além disto, foi realizada uma pesquisa bibliográfica, documental, qualitativa, em bases de dados e anais de eventos, ambos nacionais e internacionais, abordando o conceito referente a "Problema de Corte Unidimensional" que forneceu o referencial e serviu de apoio à obtenção dos resultados encontrados, em uma abordagem exploratória e qualitativa.

\section{Contexto organizacional}

\subsection{Processo de fabricação do papel}

O processo de fabricação de papel é constituído de uma cadeia de processos interligados, em algumas etapas quimicamente complexas, porém que visam, em suma, a separação de fibras e o tratamento das mesmas para que adquiram propriedades adequadas ao devido fim. Expondo, de modo simplificado, o processo executado pela Klabin, pode-se visualizar através do fluxograma na Figura 1, e logo em seguida será feita uma breve explicação a respeito das etapas de maior relevância do processo. 
FIGURA 1: PROCESSO DE PRODUÇÃO DO PAPEL CARTÃO

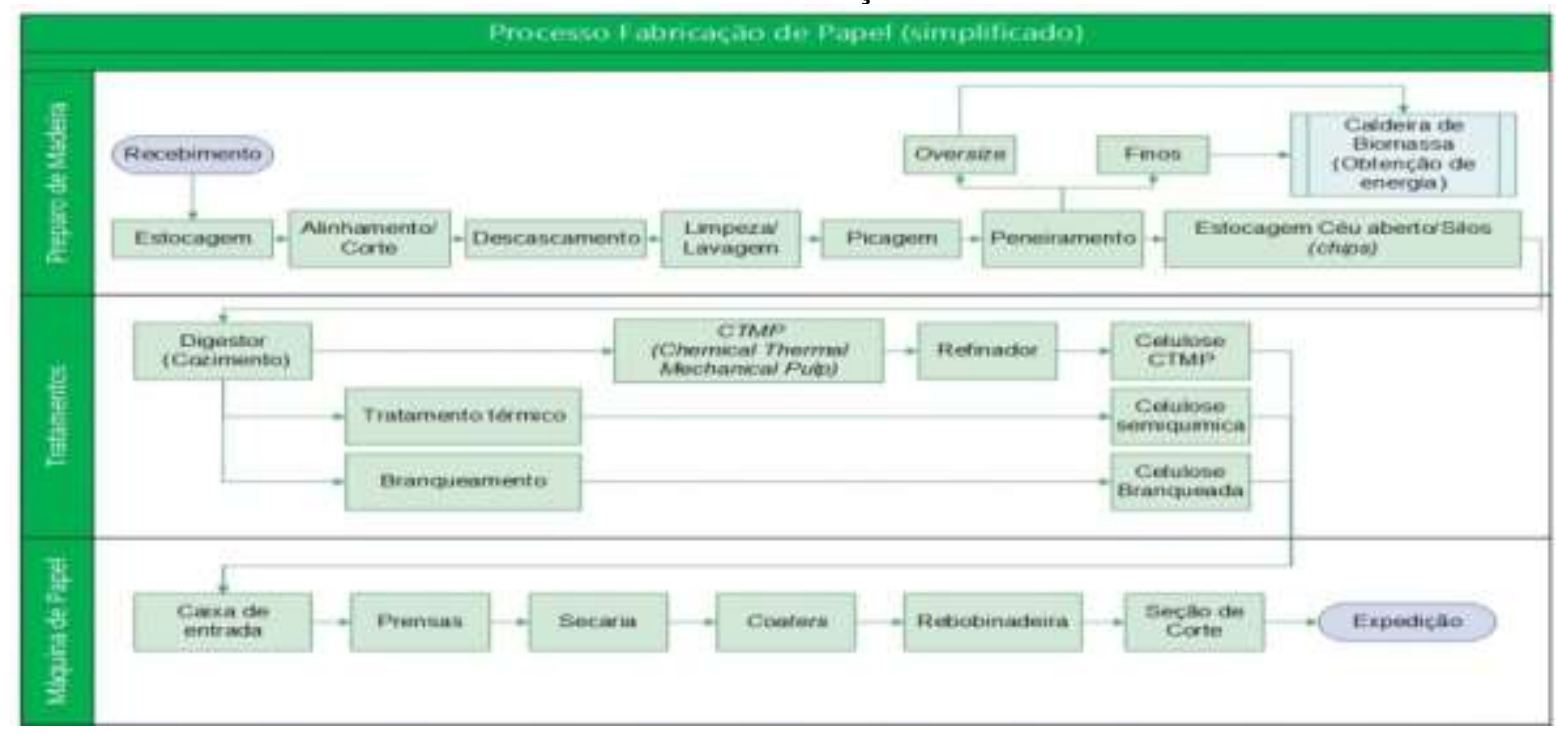

Fonte: Klabin (2013)

\subsubsection{Preparo de madeira}

Após a obtenção da madeira na área florestal, sendo elas provenientes de Pinus (hardwoodfibra longa) e Eucalipto (softwood - fibra curta), devido à necessidade de um mix de ambas para a obtenção das características desejadas ao produto final, são encaminhadas para a indústria, onde são estocadas a céu aberto, aguardando até o momento de entrar no processo, obedecendo o sistema FIFO (First In, First Out).

Em seguida a madeira passa por um descascador, o qual tem formato de um tambor, onde por atrito tanto contra as paredes como com outras toras, as cascas são retiradas. Estes resíduos de cascas são encaminhados por meio de esteiras para realizar a queima na caldeira de força, com o intuito de obtenção de energia para o processo.

Como etapa seguinte as toras de eucalipto e pinus, agora descascadas, são levadas para o picador onde será obtido o cavaco, mais comumente conhecido na indústria como chip.
Após esta etapa os chips passam por uma etapa de peneiramento, onde somente aqueles com tamanho adequado vão para a linha de produção e os rejeitos da peneira, conhecidos com finos (menores) ou oversizes (maiores), são direcionados por uma linha para reaproveitamento juntamente com as cascas, para geração de energia. Finalizando esta etapa, os chips que foram aprovados na etapa anterior vão para estocagem a céu aberto, também, onde se formam pilhas de cavaco.

\subsubsection{Tratamento térmico químico/mecânico ou CTMP (chemical thermal mechanical pulp) termoquimiomecânico)}

Nesta etapa, o cavaco, de forma simplificada, é levado ao cozimento e dependendo do tipo de tratamento que o mesmo é submetido, seu rendimento pode ser maior ou menor. Os cavacos são levados a um digestor, que possui agentes químicos como hidróxido de sódio e 


\section{ReLAlnEP}

sulfeto de sódio, à elevada temperatura, a fim de que ocorra a separação das fibras.

Como para a fabricação de papéis para embalagens é necessário que haja impressão, o papel é composto por mais de uma camada e nesse momento o processo se divide em duas linhas. De uma delas se obtém a polpa celulósica natural e da outra a polpa celulósica branqueada, que passa pelo processo químico de branqueamento.

Por fim antes de entrar na máquina de papel, as fibras recebem um tratamento de refinação, onde passando pelo refinador, as mesmas ficam com aparência "arrepiada", característica que facilita o encaixe das mesmas e também atua proporcionando uma maior resistência ao produto final.

\subsubsection{Máquina de papel}

A máquina de papel é composta por três seções importantes, das quais a caixa de entrada, onde a polpa é misturada às cargas minerais e a mistura apresenta cerca de $99,5 \%$ de água e somente $0,05 \%$ é polpa; as prensas, onde a mistura apresenta cerca de $80 \%$ de água e $20 \%$ polpa; e a secaria, onde a mistura apresenta cerca de 50\% de água e 50\% de polpa, chegando após a passagem completa pela etapa de secagem com aproximadamente $7 \%$ de umidade, característica esperada ao fim do processo. Em suma, a máquina de papel é encarregada, basicamente, de retirar a água presente na mistura.

Esta máquina possui uma mesa tripla, o que possibilita a junção das três camadas necessárias, como mostra a Figura 2, para a fabricação do produto. O "carro chefe" da unidade em estudo, da indústria Klabin é o LPB (Liquid Packaging Board), papel para embalagem de alimentos que é utilizado no Brasil e no mundo.

\section{FIGURA 2: ESTRUTURA DO PAPEL CARTÃO EM CAMADAS}

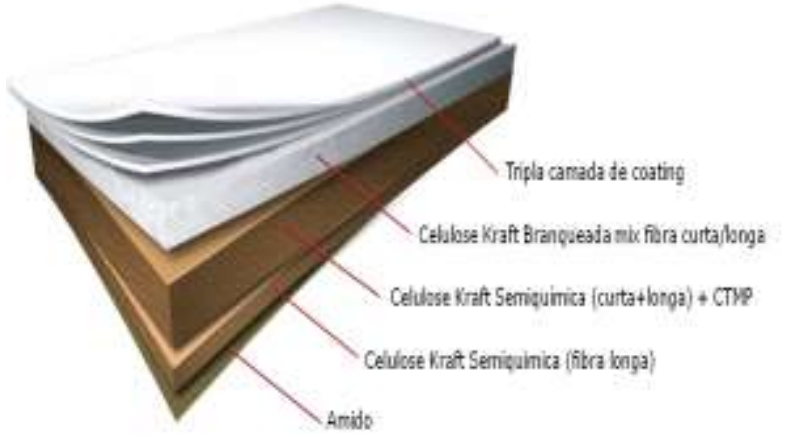

Fonte: Klabin (2013)

Como fim de todo este processo, toda máquina de papel possui ao seu final uma seção de corte como pode ser visto na Figura 3. O qual serve para que as bobinas sejam finalizadas com padrões de largura e comprimento préestabelecidos pelo cliente. Visto que as bobinas jumbo ao sair da seção de secaria, possuem 6 metros de largura e 10.000 metros de comprimento.

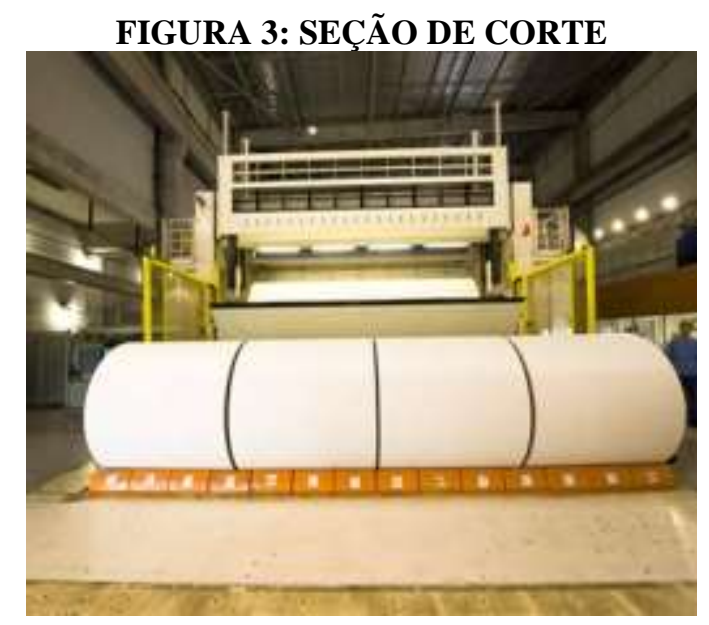

Fonte: Klabin (2013) 


\section{ReLAlnEP}

\section{Uso de Pesquisa operacional}

Muitos modelos de negócios se baseiam em ferramentas matemáticas para praticar a gestão e definir seu planejamento, bem como realizar o controle das operações e, a pesquisa operacional tem se apresentado como uma ferramenta de grande auxílio na tomada de decisão neste aspecto (CHENG; PRABHU, 2012).

Para o uso da pesquisa operacional na solução de problemas, considera-se a simplificação do problema real a fim da representação do mesmo para que se encontre uma solução ótima. Contudo, mesmo trabalhando com tal simplificação, a formulação do problema deve levar em conta as incertezas do sistema (CONGEDO; WITTEVEEN; IACCARINO, 2013).

\subsection{Problema de corte unidimensional}

Os primeiros registros de estudos relacionados ao problema de corte datam da década de 1960. Onde se identifica os pioneiros Gilmore e Gomory, tratando do problema de corte de estoques, os quais o definem como "atender um pedido a um custo mínimo para uma dimensão específica do material a ser cortado a partir de determinada dimensão de estoque a determinado custo" (GILMORE; GOMORY, 1961, p. 849).

Para Poldi e Arenales (2006, p. 473) o problema de corte unidimensional consiste em buscar "cortar objetos disponíveis para a produção de itens de modo a atender uma demanda especificada, em que apenas uma dimensão é relevante para o corte (barras, bobinas, etc.)".

$\mathrm{O}$ processo consiste em obter produtos menores a partir do corte de uma matriz com dimensões superiores, seguindo determinados padrões de corte que consistem, estes, nas variáveis de decisão (YANASSE; LAMOSA, 2007; POLDI; ARENALES, 2006). Estes problemas de corte podem ser divididos em duas categorias, de acordo com Silveira Júnior, Pinheiro e Thomaz (2004), sendo elas heurísticas e de programação linear. E assim, o uso de estratégias adequadas para os processos de corte podem guiar uma redução dos custos associados a estas atividades, bem como ao alcance de melhorias neste processo (CHERRY; ALEM JUNIOR; SILVA, 2010).

Observa-se uma grande abordagem deste problema na literatura, por Yanasse e Lamosa (2007), Poldi e Arenales (2006), Silveira Junior, Pinheiro e Thomaz (2004), Bruglieri, Maffioli e Ehrgott (2003), Cherry, Alem Junior e Silva (2010) e Suliman (2005), onde são realizadas abordagens do problema de corte unidimensional, bidimensional e integrado a estoque.

Contudo, observa-se que o problema de corte aliado a uma política de aproveitamento das sobras resultantes dessa atividade (problema de corte de estoque com sobras aproveitáveis (PCESA)) tem sido pouco estudado (CHERRY; ALEM JUNIOR; SILVA, 2010).

No presente estudo de caso, a unidade industrial em estudo realiza o aproveitamento das sobras oriundas das atividades de corte, contudo este panorama não é aqui discutido 
por não apresentar-se pertinente ao escopo do estudo.

\section{Cenário geral do problema}

Nesta seção é apresentado o problema estudado, dado o sistema considerado, as variáveis, as restrições, bem como o contexto geral que envolve toda a atividade em voga. A princípio são expostas as considerações iniciais levadas em conta para que seja possível entendimento adequado do foco e localização do problema dentro do sistema produtivo.

\subsection{Considerações iniciais}

Tendo como contexto global a produção de papel cartão, considera-se como limitação deste artigo o ambiente industrial de produção (on-site). E adota-se especificamente a etapa de corte de bobinas jumbo (material primário resultante do processo de produção do papel cartão, que sofrerá beneficiamento a partir do corte e adequação de dimensões visando atender às demandas dos clientes) para a definição do problema a ser estudado.

\subsection{Definição do problema}

Para a definição do problema e possibilidade da descoberta de uma solução factível simplifica-se, aqui, as dimensões e os diferentes tipos de bobinas comercializadas como produto final pela indústria Klabin, restringindo a quantidade de bobinas estudadas para o corte a três.

São comercializados, em prima, 3 tipos de bobinas de papel cartão (aqui definidas como sendo B1, B2 e B3). Estas bobinas são resultantes do corte de bobinas jumbo (produto primário do processo de produção do papel) com dimensões de $6 \mathrm{~m}$ de largura por $10.000 \mathrm{~m}$ de comprimento. Sabe-se também que os diferentes clientes desta unidade necessitam de diferentes tipos de bobinas e em diferentes quantidades. Observa-se, ainda, que é possível realizar diferentes cortes a partir de uma mesma matriz. Assim percebe-se, também, que ao final do corte de uma bobina jumbo pode restar uma porção que apresenta dimensões não aproveitáveis por qualquer dos clientes. Deste modo esta porção é armazenada e incorporada ao processo de produção do papel novamente fazendo parte da camada intermediária, onde não afeta a qualidade do produto. Mesmo que esta parcela de produto final não seja totalmente perdida, retornando à produção, todas as entradas (energia, mão-deobra, tempo de operação dos equipamentos, etc.) da produção inicial do mesmo não podem ser recuperadas e apresentam-se como custo adicional.

Se houver produção maior do que a demanda os produtos podem ser armazenados em estoque. Contudo esta operação deve obedecer à quantidade máxima permitida, observando que a empresa não trabalha com uma produção e venda baseada em estoques.

Além do mais considera-se, aqui, a existência de um cliente fictício (tratado aqui como Cliente C) cuja demanda é de 150.000 unidades para cada tipo de bobina (B1, B2 e B3), para que se possa observar o comportamento do modelo e a geração de resíduos frente a diferentes cenários. Esta 
característica é apenas fictícia, como colocado, não expondo uma atividade que realmente ocorre dentro da unidade, servindo apenas para trabalhar com diferentes características dentro do modelo proposto e avaliar as características da geração de sobras.

Os tipos de bobinas, suas dimensões, as demandas e as quantidades máximas de produção, levando em conta o estoque máximo permitido na unidade industrial em estudo são apresentados na Tabela 1 a seguir.

TABELA 1: DEMANDA MENSAL E ESTOQUE MÁXIMO DOS TIPOS DE BOBINAS PRODUZIDAS NA UNIDADE FABRIL (EM UNIDADES DE BOBINAS)

\begin{tabular}{c|ccc}
\hline $\begin{array}{c}\text { Tipo de } \\
\text { bobina }\end{array}$ & B1 & B2 & B3 \\
\hline Demanda & 1.500 .000 & 750.000 & 850.000 \\
$\begin{array}{c}\text { Estoque } \\
\text { máximo }\end{array}$ & 100.000 & 250.000 & 250.000 \\
$\begin{array}{c}\text { Demanda }+ \\
\text { Estoque } \\
\text { máximo }\end{array}$ & 1.600 .000 & 1.000 .000 & 1.000 .000 \\
\hline
\end{tabular}

Deste modo pode-se observar que a política de estoque restringe de certa forma a produção das bobinas, uma vez que deve ser respeitado o espaço disponível para o armazenamento de excedentes.

Outra informação indispensável é a quantidade e proporção do pedido do cliente C, apresentadas na Tabela 2 .

TABELA 2: PEDIDO DO CLIENTE C (EM UNIDADES DE BOBINAS)

\begin{tabular}{c|ccc}
\hline Tipo de bobina & B1 & B2 & B3 \\
\hline $\begin{array}{c}\text { Pedido do Cliente } \\
\text { C }\end{array}$ & 150.000 & 150.000 & 150.000 \\
\hline
\end{tabular}

É necessário observar que estas quantidades já estão incluídas na demanda mensal global; a
Tabela 2 apenas especifica as quantidades que são demandadas pelo Cliente C. Supõe-se que este pedido ocorre sempre na mesma quantidade e na mesma proporção (para cada B1 são necessárias 1 B2 e 1 B3). Além do mais, esta proporção define exatamente as proporções utilizadas no padrão de corte $\mathrm{X} 3$, que será apresentado na seção seguinte, tornando imperativo que a produção deste padrão de corte siga na produção em paralelo com a demanda do referido cliente em detrimento do pedido necessitar ser atendido de forma rápida.

\subsubsection{Padrões de cortes}

Para a realização dos cortes é necessário levar em consideração as dimensões da bobina jumbo. As Bobinas B1, B2 e B3 possuem diferentes dimensões quanto à largura, como mostrado na Tabela 3. Contudo, não diferem no comprimento, tendo todas o mesmo comprimento da bobina jumbo (10.000 m), fazendo, então, com que no problema tenha de ser considerada apenas uma dimensão para os cortes, utilizando um panorama unidimensional.

TABELA 3: DIMENSÕES DOS TIPOS DE BOBINAS

\begin{tabular}{c|ccc}
\hline & & $\begin{array}{c}\text { Largura } \\
(\mathbf{m})\end{array}$ & $\begin{array}{c}\text { Comprimento } \\
(\mathbf{m})\end{array}$ \\
\hline $\begin{array}{l}\text { Tipo de } \\
\text { bobina }\end{array}$ & B1 & 2,4 & 10.000 \\
& B2 & 1,8 & 10.000 \\
& B3 & 1,4 & 10.000 \\
\hline
\end{tabular}

Assim, considerando que os cortes realizados não podem exceder as dimensões da bobina jumbo, observa-se que são possíveis algumas 


\section{ReLAInEPY}

combinações de corte que atendem a esta abrangem todos os tipos de bobinas restrição. Deste modo são apresentados na Tabela 4 os padrões de corte praticáveis e ao final, os respectivos resíduos gerados (largura(m)) por cada combinação.

De acordo com estas informações, evidencia-se que seria preferível utilizar apenas os padrões ofertados e são os padrões que geram as menores quantidades de resíduos. Contudo, é necessário que sejam atendidas todas as restrições do sistema em relação à demanda e estoque máximo.

$\mathrm{X} 2$, X3 e X8, uma vez que estes, juntos,

TABELA 4: PADRÕES DE CORTE, DE ACORDO COM COMBINAÇÕES DE BOBINAS, E SEUS RESÍDUOS

\begin{tabular}{c|cc}
\hline Padrão de corte & Combinação dos tipos de bobinas resultantes & Resíduo gerado (largura(m)) \\
\hline X1 & $\mathrm{B} 1+\mathrm{B} 1$ & 1,2 \\
X2 & $\mathrm{B} 1+\mathrm{B} 2+\mathrm{B} 2$ & 0 \\
X3 & $\mathrm{B} 1+\mathrm{B} 2+\mathrm{B} 3$ & 0,4 \\
X4 & $\mathrm{B} 1+\mathrm{B} 3+\mathrm{B} 3$ & 0,8 \\
X5 & $\mathrm{B} 2+\mathrm{B} 2+\mathrm{B} 2$ & 0,6 \\
X6 & $\mathrm{B} 2+\mathrm{B} 2+\mathrm{B} 3$ & 1 \\
X7 & $\mathrm{B} 2+\mathrm{B} 3+\mathrm{B} 3$ & 1,2 \\
X8 & $\mathrm{B} 3+\mathrm{B} 3+\mathrm{B} 3+\mathrm{B} 3$ & 0,4 \\
\hline
\end{tabular}

$\mathrm{Na}$ seção seguinte são traçadas algumas considerações a respeito da aplicação do modelo no alcance de uma solução factível para o problema de estudo.

\subsection{Considerações a respeito do estabelecimento do problema}

Como descrito na seção 1, objetiva-se formular um modelo de programação linear de modo a buscar a redução da porção de papel a descartar, em um mês de operação de uma unidade industrial da empresa Klabin, atendendo às restrições de produção impostas.

De qualquer modo deve-se salientar que as características aqui expostas representam uma simplificação das características reais do processo em questão. Uma vez que os volumes considerados de demanda, estoques, resíduos, tempo de operação da máquina (mesmo que aqui não contabilizados, mas que influenciam no resultado final da produção), fluxo de produtos, etc., podem não ser fidedignas aos dados reais para um período específico.

As possíveis discrepâncias podem ocorrer devido às características peculiares de mercado e situação das instalações; principalmente o volume de estoques, uma vez que, como já exposto, a unidade não trabalha com produção e venda baseadas em estoques, em prima devido ao enorme fluxo de entrada e saída de matéria-prima e produto acabado. 


\section{ReLAInEP}

\section{Exposição do modelo matemático} proposto e solução do problema

A seguir é apresentada a solução ótima encontrada para o problema considerado, utilizando a ferramenta LINGO.

São estudados três cenários em busca da solução do problema:

- Cenário 1: Modelo com as restrições de demanda, estoque máximo e Cliente $\mathrm{C}$;

- Cenário 2: Modelo com as restrições de demanda e estoque máximo;

- Cenário 3: Modelo com as restrições de demanda.

\subsection{Estabelecimento do modelo de programação linear (PL)}

Como apresentado na definição do problema tem-se uma matriz jumbo de comprimento igual a 6 metros que precisa ser cortada de acordo com padrões de corte i de modo a obter os produtos $\mathrm{j}$.

Assim, estabelece-se:

- os padrões de corte

Corte $(i)^{T}=\left[\begin{array}{llllllll}X 1 & X 2 & X 3 & X 4 & X 5 & X 6 & X 7 & X 8\end{array}\right]$ , sendo, para o padrão de corte $X 1$, até para $\mathrm{X} 8$, definindo os padrões de corte a serem praticados, os quais representam as variáveis do problema, cujos coeficientes serão determinados pela solução do problema e definirão as quantidades a serem produzidas;

- os tipos de bobinas
$\operatorname{Bobinas}(j)=\left[\begin{array}{lll}B 1 & B 2 & B 3\end{array}\right]$, sendo, para o tipo de bobina B1, até para o tipo B3, definindo os recursos.

- as quantidades de resíduos gerados pelo uso de cada padrão de corte

$\operatorname{Resíduos}(i)^{T}=\left[\begin{array}{llllllll}1,2 & 0 & 0,4 & 0,8 & 0,6 & 1 & 1,2 & 0,4\end{array}\right]$ , definindo o resíduo resultante do uso do respectivo padrão de corte.

- a matriz que define a participação das nos

Recurso $(i, j)=\left[\begin{array}{lll}2 & 0 & 0 \\ 1 & 2 & 0 \\ 1 & 1 & 1 \\ 1 & 0 & 2 \\ 0 & 3 & 0 \\ 0 & 2 & 1 \\ 0 & 1 & 2 \\ 0 & 0 & 4\end{array}\right]$

Deste modo, analisando as restrições, pode-se ainda, definir o vetor em função da demanda mínima necessária e o vetor (ambos em unidades) para atender os clientes da unidade no período considerado, como segue:

$\begin{aligned} \text { MinDemanda }(j) & =\left[\begin{array}{lll}1.500 .000 & 750.000 & 850.000\end{array}\right] \\ \text { MaxEstoque }(j) & =\left[\begin{array}{lll}500.000 & 250.000 & 150.000\end{array}\right]\end{aligned}$

Onde a soma destes vetores (MinDemanda(j) + MaxEstoque(j)) representa a quantidade máxima a ser produzida das bobinas B1, B2 e B3, a fim de respeitar estas restrições do sistema.

Em posse de tais informações, pode-se definir a função objetivo, visando minimizar os 
resíduos gerados pelas atividades de corte, obtendo (Equação 1).

Minimizar $=\sum_{i=1}^{i}[\operatorname{Resíduos}(i) * \operatorname{Corte}(i)](1)$

De modo que a função objetivo atenda às seguintes restrições:

$\left[\sum_{i=1}^{i} \operatorname{Recurso}(i, j) * \operatorname{Corte}(i)\right] \geq \operatorname{MinDemanda}(j), \quad \mathrm{em}$ função da demanda mínima.

$\left[\sum_{i=1}^{i} \operatorname{Recurso}(i, j) * \operatorname{Corte}(i)\right] \leq[\operatorname{MinDemanda}(j)+\operatorname{MaxEstoque}(j)]$ , em função do estoque máximo.
Corte(3) $\geq 150.000$ em função do atendimento do Cliente $\mathrm{C}$.

\subsubsection{Cenário 1}

Seguindo o modelo de programação apresentado, por meio da ferramenta LINGO obteve-se a solução ótima do problema, exposta na Tabela 5.

TABELA 5: QUANTIDADE A SER PRODUZIDA DE CADA TIPO DE BOBINA APLICANDO-SE A SOLUÇÃO ÓTIMA - CENÁRIO 1

\begin{tabular}{l|ccccc}
\hline & & X1 (un) & X2 (un) & X3 (un) & Total (un) \\
\hline Quantidade do padrão de corte & & 287.500 & 75.000 & 850.000 & \\
Quantidade de bobinas por tipo & B1 & 575.000 & 75.000 & 850.000 & 1.500 .000 \\
& B2 & - & 150.000 & 850.000 & 1.000 .000 \\
& B3 & - & - & 850.000 & 850.000 \\
\hline
\end{tabular}

Fonte: Elaborado pelos autores

Observa-se que dadas as restrições de estoque máximo, demanda e, com atenção especial, a restrição do uso do padrão X3 para atender o Cliente $\mathrm{C}$, a solução ótima prevê o uso de apenas 3 dos padrões de corte, sendo eles X1, X2 e X3.

Foi obtido, portanto, que a produção de 1.500 .000 de B1, 1.000 .000 de B2 e 850.000 de B3 é a proporção que minimiza a geração de resíduos, atendendo às restrições de demanda e estoque existentes, resultando em um resíduo total com dimensões de $685.000 \mathrm{~m}$ de largura por $10.000 \mathrm{~m}$ de comprimento.

\subsubsection{Cenário 2}

Pode-se considerar, também, um resultado que leve em conta a exclusão da restrição da necessidade de atendimento da demanda do cliente $\mathrm{C}$ com o uso exclusivo do padrão de corte X3.

Assim tem-se que o modelo encontraria uma solução com uma geração de resíduos idêntica à do Cenário 1. Contudo os padrões de corte utilizados são diferentes. São utilizados, agora, os padrões X1, X2 e X4. Realizando a proporção adequada, de cada padrão com os tipos de bobinas obtidas, tem-se os resultados mostrados na Tabela 6. 
TABELA 6: QUANTIDADE A SER PRODUZIDA DE CADA TIPO DE BOBINA APLICANDO-SE A SOLUÇÃO ÓTIMA - CENÁRIO 2

\begin{tabular}{c|ccccc}
\hline & & X1 (un) & X2(un) & X4(un) & Total (un) \\
\hline Quantidade do padrão de corte & & 287.500 & 500.000 & 425.000 & \\
Quantidade de bobinas por tipo & B1 & 575.000 & 500.000 & 425.000 & 1.500 .000 \\
& B2 & - & 1.000 .000 & - & 1.000 .000 \\
& B3 & - & - & 850.000 & 850.000 \\
\hline
\end{tabular}

Fonte: Elaborado pelos autores

A solução que otimiza, neste caso, a geração mínima de resíduos em detrimento das atividades de corte ordena a produção de 1.500.000 de bobinas do tipo B1, 1.000.000 do tipo B2 e 850.000 B3 resultando em um total de resíduos de $685.000 \mathrm{~m}$ de largura por $10.000 \mathrm{~m}$ de comprimento.

Observa-se, desta forma que o Cenário 2 gera uma quantidade de resíduos igual à quantidade obtida pela solução ótima no Cenário 1. Mostrando que mesmo com parâmetros diferentes que restringem a produção, não sendo imperativo neste caso que se utilize o padrão de corte $\mathrm{X} 3$ para atender à demanda de um determinado cliente. O resultado obtido quanto à geração de resíduos é o mesmo, e mesmo as quantidades de acordo com os tipos de produto final (B1, B2 e B3) não diferem.

\subsubsection{Cenário 3}

Em um panorama menos restritivo pode-se considerar, a partir do Cenário 2, a exclusão da política de estoque máximo, onde a solução ótima, neste caso, prevê a utilização de apenas dois dos padrões de corte possíveis, sendo os que resultam em uma menor geração de resíduos e abrangem todos os tipos de bobinas que precisam ser produzidas. Contudo, desta maneira há uma produção excessiva de bobinas do tipo B2, como evidenciado na solução e mostrado na Tabela 7.

\section{TABELA 7: QUANTIDADE A SER PRODUZIDA DE CADA TIPO DE BOBINA APLICANDO-SE A SOLUÇÃO ÓTIMA, EXCETO A RESTRIÇÃO DO CLIENTE C E A POLÍTICA DE ESTOQUE} MÁXIMO - CENÁRIO 3

\begin{tabular}{c|cccc}
\hline & & X2 (un) & X8 (un) & Total (un) \\
\hline Quantidade do padrão de corte & & 1.500 .000 & 212.500 & \\
Quantidade de bobinas por tipo & B1 & 1.500 .000 & - & 1.500 .000 \\
& B2 & 3.000 .000 & - & 3.000 .000 \\
& B3 & - & 850.000 & 850.000 \\
\hline
\end{tabular}

Fonte: Elaborado pelos autores

Este modelo, pouco restrito, faz com que a produção de bobinas tipo B2, atendendo apenas às restrições de demanda da unidade, gere uma enorme quantidade de estoque, o que pode acarretar em alto custo para permanência dos produtos nas instalações. Por outro lado, pode-se considerar uma produção menor, ou mesmo nula, deste tipo de produto em períodos próximos. Todavia esta operação se mostraria 
como uma nova restrição, merecendo um estudo mais específico de sua influência.

Em linhas gerais, apesar desta produção excessiva, o objetivo do modelo foi alcançado satisfatoriamente, obtendo um resíduo total com dimensões de $85.000 \mathrm{~m}$ de largura por $10.000 \mathrm{~m}$ de comprimento, considerando o comprimento da matriz utilizada para os cortes.

\section{Considerações finais e sugestões para estudos futuros}

O planejamento do modo de realizar a produção é fator diferencial no andamento de uma empresa, seja referente à política financeira, ambiental ou de qualquer âmbito.

O objetivo inicial deste trabalho, de minimizar os resíduos resultantes das atividades de corte visando à adequação de um produto primário aos padrões de comercialização, foi alcançado com êxito. Conclui-se que uma sobra superficial final de dimensões $685.000 \mathrm{~m}$ por $10.000 \mathrm{~m}$ é a solução ótima do problema de corte unidimensional tratado neste estudo de caso, de forma a atender a todas as restrições inicialmente estabelecidas.

Considerando-se outros cenários de avaliação, menos restritivos, pode-se obter uma sobra superficial de $85.000 \mathrm{~m}$ por $10.000 \mathrm{~m}$, contudo de forma a atender apenas a demanda de produtos acabados da unidade de forma mais flexível.

A política de ação da empresa em estudo impera que estas sobras sejam reaproveitadas, sendo armazenadas e voltando ao processo de produção para fazer parte da camada intermediária do papel. Todavia, não é discutida e devidamente exposta tal prática neste trabalho, uma vez que o objetivo do estudo possui outro foco.

Em estudo futuros sugere-se levar em consideração a integração da etapa de corte com todas as outras etapas do processo de produção. Uma vez que da interação entre todas as etapas pode ser possível obter uma solução que melhor se adéque ao perfil da empresa e às características da produção de uma forma mais abrangente e que seja menos onerosa.

\section{Referências}

BRUGLIERI, Maurizio; MAFFIOLI, Francesco; EHRGOTT, Matthias. Cardinality constrained cut problems: complexity and algorithms. Discrete Applied Mathematics, v. 137, p. 311-341. 2004.

CHENG, Chen-Yang; PRABHU, Vittal. Evaluation models for service oriented process in spare parts management. Journal of Intelligent Manufacturing, n. 23, p. 1403-1417. 2012.

CHERRY, Adriana Cristina; ALEM JUNIOR, Douglas Jose; SILVA, Ivan Nunes. Inferência fuzzy para o problema de corte de estoque com sobras aproveitáveis de material. Pesquisa Operacional, v. 31, n. 1, p. 173-194. 2011.

CONGEDO, Pietro Marco; WITTEVEEN, Jeroen; IACCARINO, Gianluca. A simplex-based numerical framework for simple and efficient robust design optimization. Computational Optimization and Applications, v. 56, p. 231-251. 2013.

FIGUEIRA, Gonçalo; SANTOS, Maristela Oliveira; ALMADA-LOBO, Bernardo. A hybrid VNS approach for the short-term productions planning and scheduling: A case study in the pulp and paper industry. Computers and Operations Research, v. 40, p. 1804-1818. 2013.

GILMORE, P.C.; GOMORY, R.E.. A linear 
programming approach to the cutting stock 2007. problem. Operations Research, v. 9, n.6, p. 848-859. 1961.

GILMORE, P.C.; GOMORY, R.E.. A linear programming approach to the cutting stock problem - Part II. Operations Research, v. 11, n.6, p. 863-888. 1963.

\section{KLABIN, - Formulário de \\ Referência - 2013 - Klabin S.A.. Acesso em 27/08/2013. Disponível em: <http:/ / klabin.infoinvest.com.br/ptb/2057/13 69425128_235494570_html/1369425128_235 494570.html>}

KAMIMURA, Quésia Postigo. PAES, Evandro da Silva; OLIVEIRA, Edson Aparecida Araujo Querido. Inovação Tecnológica: início de novos negócios em TI - estudo de caso numa empresa de bens de capital do Vale do Paraíba. Latin American Journal of Business Management, v. 3, n. 2, p. 174-187, juldez/2012, Taubaté, SP, Brasil.

LUZ, João Augusto Ferreira; OLIVEIRA, Edson Aparecida de Araújo Querido; SANTOS, Vilma da Silva; QUINTAIROS, Paulo Cesar Ribeiro. Inovação Tecnológica de produtos e processos na indústria automobilística. Latin American Journal of Business Management, v. 3, n. 2, p. 210-225, jul/dez2012, Taubaté, SP, Brasil.

POLDI, Kelly Cristina; ARENALES, Marcos Nereu. Heurística para o problema de corte de estoque unidimensional inteiro. Pesquisa Operacional, v. 26, n. 3, p. 473, 492. 2006.

SILVERA JÚNIOR, José Aélio; PINHEIRO, Plácido Rogério; THOMAZ, Antonio Clécio Fontanelle. Otimização das perdas em cortes guilhotinados para bobinas de aço na indústria metalmecânica. In: Simpósio Brasileiro de Pesquisa Operacional, 36. O impacto da Pesquisa Operacional nas novas tendências multidisciplinares. São João Del Rei, Brasil. Anais. Minas Gerais, Brasil. 2004.

SULIMAN, S. M. A.. A sequential heuristic procedure for the two-dimensional cutting-stock problem. International Journal of Production Economics, v. 99, p. 177-185. 2006.

YANASSE, Horacio Hideki; LAMOSA, Maria José Pinto. An integrated cutting stock and sequencing problem. European Journal of Operational Research, v. 183, p. 1353-1370. 